\title{
ANÁLISIS TECNOLÓGICO DE LOS MONOLITOS DE PIEDRA VERDE HALLADOS EN LA PLAZA DE LA PIRÁMIDE DE LA LUNA, TEOTIHUACÁN ${ }^{8}$
}

\author{
Technological analysis of Green Stone Monoliths found at Plaza of \\ the Moon Pyramid, Teotihuacán
}

Verónica Ortega Cabrera9, Viridiana Guzmán Torres10, Emiliano R. Melgar Tísoc11 y Serafín Sánchez Pérez ${ }^{12}$

\section{Resumen}

Durante las excavaciones realizadas en el "Proyecto de conservación Integral de Edificio 1, Plaza de la Pirámide de la Luna, Teotihuacán, temporada 2014" y "Proyecto Plaza de la Pirámide de la Luna, Estructura A. (Quincunce), temporada 2015", fue posible la recuperación de 5 monolitos de piedra verde. Para resolver cuestiones como qué tipo de materia prima se utilizó para su manufactura de los monolitos y qué herramientas fueron predilectas para la elaboración de los mismos; para ello fue necesario proponer una metodología idónea para el análisis, ya que debido a las dimensiones y peso de los mismos limitaba la transportación a un laboratorio para su estudio. Para conocer la tecnología fue necesario identificar la materia prima por medio de análisis de composición química por Microsonda de Rayos X (EDS) y micro-Raman ( $\mu$ Raman). En cuanto a las huellas de manufactura fueron caracterizadas con arqueología experimental y microscopía electrónica de barrido (SEM). Se encontró que la manufactura fue local utilizando herramientas como pedernales y lajas de andesita; en cuanto el tipo de materia prima se identificó como piedras verdes metamórficas con distintas variaciones en sus elementos entre ellos el cuarzo y un parecido con las serpentinas.

Palabras clave: EDS, SEM, $\mu$ Raman, Arqueología experimental, huellas de manufactura, Teotihuacan- Plaza de la Luna.

\footnotetext{
Abstract:

${ }^{8}$ Recibido 27/11/2018. Aceptado 14/12/2018. http://doi.org/10.15366/baexuam2018-19.13.003

${ }^{9}$ Subdirección Técnica de la Zona de Monumentos Arqueológicos de Teotihuacan. ORCID: 0000-0002-72152228. veronicabmx@yahoo.com.mx

${ }^{10}$ Escuela Nacional de Antropología e Historia, Museo Nacional de Antropología e Historia. ORCID: 00000003-2070-1843 viridianaguz23@gmail.com

${ }^{11}$ Museo del Templo Mayor. ORCID: 0000-0002-7300-3224. anubismarino@gmail.com

${ }^{12}$ Laboratorio de Suelos y Sedimentos de la Escuela Nacional de Antropología e Historia. ORCID: 0000-00016324-9493 serass1@yahoo.com.mx
} 
V. Ortega, V. Guzmán, E. R. Melgar y S. Sánchez

Análisis tecnológico de los monolitos de piedra verde hallados en la Plaza de la Pirámide de la Luna, Teotihuacán

During the excavations carried out in 2014 and 2015 in the Plaza of the Moon Pyramid in Teotihuacan, Mexico, five monoliths made out of green stone were recovered. In order to identify the raw material and the tools used for their manufacture, a suitable methodology had to be proposed since the monoliths' dimensions and weight greatly limited their transportation to a laboratory for analysis. The raw materials were identified through chemical composition analyses using X-ray microprobe and micro-Raman. Manufacture prints were characterized by experimental archaeology and scanning electron microscopy. We found that the monoliths were manufactured locally using flint and andesite tools; the raw materials were identified as green metamorphic stones with varying elemental composition, including quartz, and resembling serpentines.

Key words: EDS, SEM, $\mu$ Raman, Experimental archeology, manufacturing, TeotihuacanPlaza de la Luna.

\section{INTRODUCCIÓN}

Tierra pedazo de gloria dueña de una historia hermosa y cabal.

(Fernando Moncada)

En el pasado de la humanidad han existido Ciudades que con su historia han dejado tal brillo y esplendor que aún es persistente hasta nuestros días para ser admiradas y estudiadas; ejemplos son ciudades como Roma, Constantinopla y Alejandría (Manzanilla, 2012:17)

Una de las grandes ciudades enigmáticas en el contexto de la historia prehispánica de México, resurgió cuando los mexicas (1325-1521 d.n.e) la descubrieron y nombraron como "Teotihuacan"; se encuentra ubicada a poco más de $45 \mathrm{~km}$ al noroeste de la actual Ciudad de México y aproximadamente a 2,275 msnm, y actualmente es considerada como Patrimonio de la Humanidad por la UNESCO. Los antiguos vestigios son imagen del gran desarrollo que alcanzó a medidos del primer milenio de nuestra era y fue abandonada casi por completo en el siglo VII. Su prestigio fue tal que aun abandonada y extinta la sociedad, los mexicas la consideraron como el lugar en donde se crearon el sol y la luna (Graulich, 2014:25).

La antigua metrópoli tenía a su disposición manantiales de agua dulce, materiales volcánicos (escoria volcánica, andesita basalto, toba volcánica) para la construcción de arquitectura, fabricación de ornamentos, objetos votivos, herramientas por mencionar algunos usos. Contó con yacimientos de materias primas cercanos como son las de obsidiana de Otumba y la Sierra de las Navajas (Manzanilla, 2012:18). 


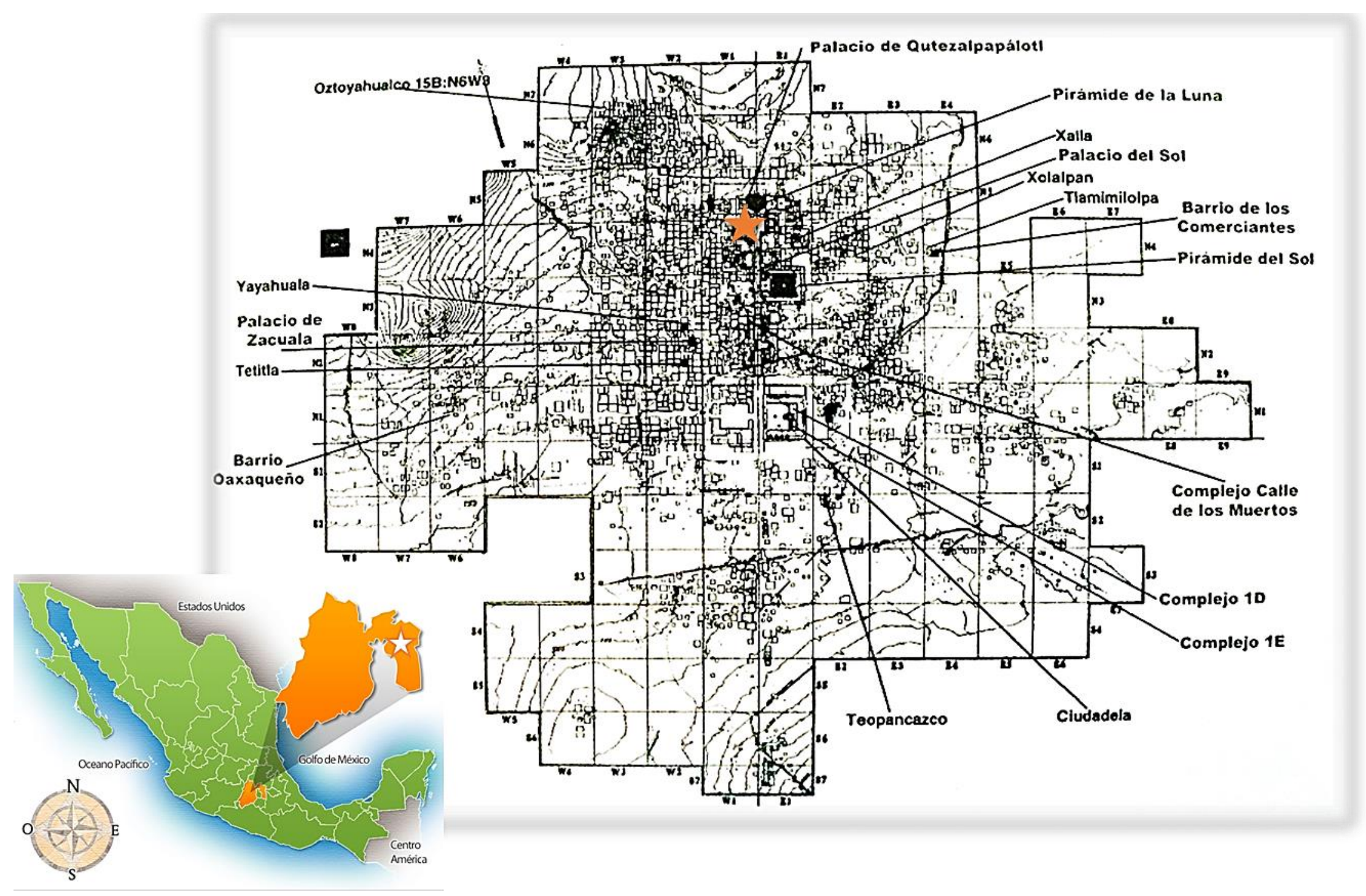

Figura 1. Ubicación de la Plaza de la Pirámide de la Luna en la Ciudad de Teotihuacan (Tomado de Manzanilla, 2012:19). 
V. Ortega, V. Guzmán, E. R. Melgar y S. Sánchez

Análisis tecnológico de los monolitos de piedra verde hallados en la Plaza de la Pirámide de la Luna, Teotihuacán

De acuerdo con la propuesta de Linda Manzanilla, la ciudad debió dividirse en cuatro sectores, que funcionaron como polos administrativos del Estado (Ibídem, 20) sin embargo existen otras ideas al respecto y la discusión aún sigue abierta.

Es justo mencionar, que, si bien se han realizado en sitio distintas investigaciones, sobresale el trabajo realizado por René Millon de la Universidad de Rochester con el "Teotihuacan Mapping Project" y sus colaboradores Bruce Drewitt, James Bennyhoff y George Cowgill en 1973, el cual consistió en el levantamiento topográfico detallado de los vestigios de la antigua urbe, con ayuda de fotografías aéreas y recorridos de superficie. El resultado fue el plano arqueológico y topográfico del asentamiento, que continúa siendo la base de los estudios en superficie y de excavación (Fig. 1).

\section{EL PROYECTO DE CONSERVACIÓN INTEGRAL PLAZA DE LA LUNA, 2014}

La Plaza de la Luna no había tenido intervenciones en materia de conservación arqueológica, prácticamente desde que fue despejada y abierta a la visita pública en la década de los años sesenta del siglo pasado. Hacia el año 2014 diversos edificios ya presentaban malas condiciones de conservación, mismas que ponían en riesgo la estabilidad de los basamentos y la seguridad de los visitantes. Por tal motivo se emprendió un proyecto de conservación integral, bajo la dirección de la Dra. Verónica Ortega Cabrera, que partió de un diagnóstico general tanto de los edificios como de la superficie de la plaza, con la idea de identificar posibles zonas de inestabilidad en el terreno que pudiesen ser la causa de algunas fracturas y grietas en los cuerpos de los edificios 1 y 7.

Por tal motivo se aplicaron técnicas de prospección eléctrica, con el apoyo de la Coordinación Nacional de Arqueología del INAH, quien comisionó al Arqueólogo Osvaldo Esterpone para que llevara a cabo dichos trabajos. La prospección se concentró en el Edificio 1 y en la Estructura A, esta última conocida también como "Quincunce"; obteniendo imágenes en las que se identificaron diversas anomalías en el subsuelo. En el año 2016, se llevaron a cabo excavaciones arqueológicas para identificar la naturaleza de las anomalías detectadas, lo que dio como resultado el hallazgo de un sistema de fosas excavadas en el tepetate (roca madre) en tiempos prehispánicos.

Tres fosas excavadas de poniente a oriente, de $2 \mathrm{~m}$ de diámetro por $2.50 \mathrm{~m}$ de profundidad en promedio, se localizaron en la entrada del edificio, mientras que en lado poniente fue excavada una enorme fosa de silueta trilobulada que alcanzó los $3.50 \mathrm{~m}$ de profundidad. Además, en el sector sur de la estructura A se halló una fosa más, así como una serie de pequeñas oquedades distribuidas de manera aleatoria en gran parte de la superficie de los pisos. En el interior de algunas de las fosas había monolitos de piedra verde, colocados en posición vertical, recargados en las paredes; la altura de los monolitos varía de $1.25 \mathrm{~m}$ a 1.50 m y su peso oscila entre los 200 y los $300 \mathrm{~kg}$ (Fig. 2). El hallazgo de estos objetos generó una serie de preguntas en torno a la función tanto de las fosas como de ellos mismos, por lo que se consideró indispensable identificar su materia prima y las técnicas de manufactura, con el objetivo de establecer su procedencia y una posible correspondencia con los patrones de trabajo de los objetos identificados para Teotihuacan. 
Existen varias propuestas para resolver la interrogante de cómo fueron elaborados algunos objetos encontrados en el área de Teotihuacan. Julie Gazzola (2009:61) propone, a través de cadenas operativas, la manufactura de máscaras teotihuacanas, estableciendo una serie de pasos que probablemente fueron seguidos para obtener la forma de las máscaras. Asi mismo, realza la importancia de tomar en cuenta los contextos para proponer herramientas para la manufactura de los objetos lapidarios. Sin embargo, sus propuestas de qué herramientas debieron ser utilizadas para la manufactura de las máscaras son hipotéticas y apoyadas en la proximidad espacial de algunos instrumentos de trabajo; valiosas en sí, pero requieren comparaciones de las huellas de manufactura y de uso para confirmarlas de forma concluyente.

En trabajos recientes en el área de Teotihuacan; Emiliano Melgar, Reyna Solís y Ruvalcaba Sil (2012) mencionan la carencia de evidencias arqueológicas directas para saber, en un primer momento, con qué herramientas fueron elaborados los objetos. Plantean que a través de arqueología experimental y análisis de huellas de manufactura es posible identificar dichas herramientas, así como comparando huellas con otras catalogadas de estilo teotihuacano, lo que permite dilucidar qué objetos son locales y cuales foráneos. Así mismo, los análisis de composición elemental remiten al área de extracción de los materiales por lo que, en teoría, permiten entender el uso de materiales locales o foráneos para la producción de artefactos e intercambio de los mismos. Sin embargo, los autores reconocen que es necesario seguir con este tipo de trabajos en el área para poder caracterizar las variantes en materias primas, morfología y tecnología. 


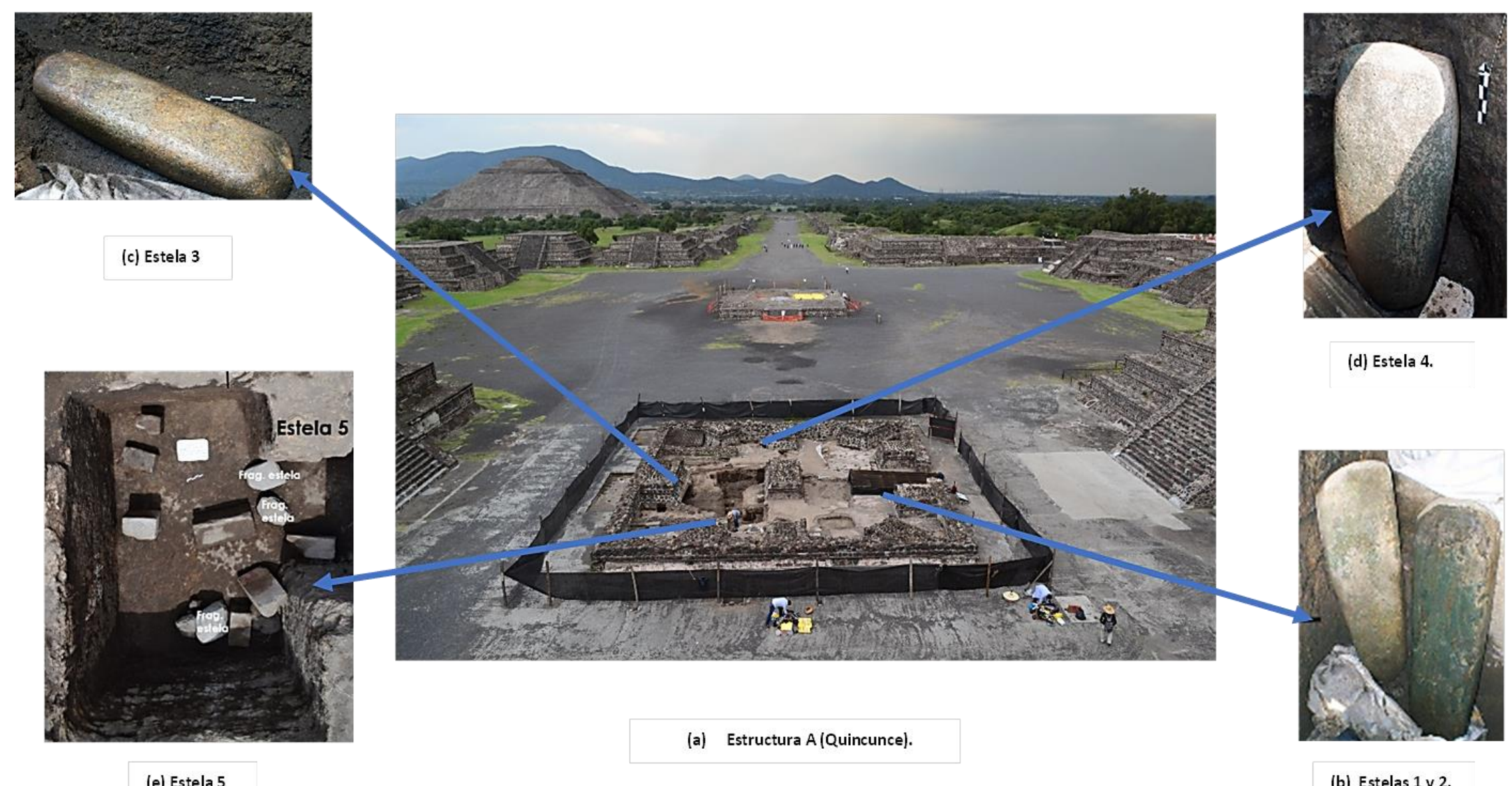

(e) Estela 5

(b) Estelas 1 y 2 .

Figura 2. (a) Estructura A (Quincunce), Plaza de la Pirámide de la Luna, (b) Estela 1 y 2, (c) Estela 3, (d) Estela 4 y (e) Tres fragmentos de la estela 5. (Fotografías de Aldo Díaz Avelar para el Proyecto de Conservación Integral Plaza de la Luna, 2014 y 2015). 


\section{METODOLOGÍA}

Debido al tamaño y peso de los monolitos, fue preciso plantear una metodología óptima para su estudio, la cual se describe a continuación:

\subsection{Toma de muestra}

Con la asesoría y colaboración del Doctor Serafín Sánchez Pérez responsable del Laboratorio de Suelos y Sedimentos de la Escuela Nacional de Antropología e Historia (ENAH) en la Ciudad de México, se realizó la toma de muestra para los análisis de composición elemental de cada monolito, misma que consistió en un fragments de aproximadamente de $1 \mathrm{~cm}$ de ancho por $1 \mathrm{~cm}$ de largo, tomando en cuenta las superficies aptas para realizar el muestreo y evitar la afectación a los objetos (Fig. 3).

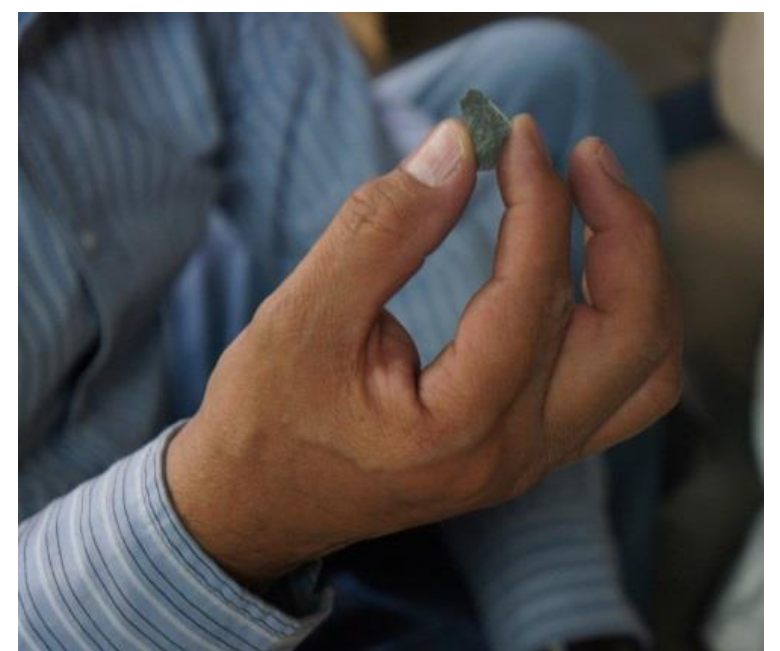

Figura 3. Toma de muestra de estela (Fotografía de Viridiana Guzmán, 2017). 


\subsection{Análisis de energía dispersa de rayos $\mathrm{x}$ (EDS)}

Con el análisis de la Microsonda de Rayos X o Espectroscopía de Dispersión de Energía de Rayos X (EDS), es posible obtener información sobre la composición elemental de manera semicuantitativa (\% en peso y $\%$ atómico) de las piezas a través de detectores de rayos $\mathrm{X}$ (Fig. 4). Con ello se pueden identificar los elementos químicos con número atómico mayor a 5 de la tabla periódica presentes en las piezas, gracias a la detección de la energía resultante de la transición de los electrones excitados de cada átomo al ser ionizados por el haz primario de electrones (Kang, 2013:663). El equipo EDS utilizado, marca INCA, se encuentra en el Laboratorio de Microscopía Electrónica del INAH. Para el análisis se emplearon electrones retrodispersados (BES) y el tiempo de adquisición fue de 120 segundos. Este análisis a piezas se realizó con el apoyo técnico del Mtro. Mario Monroy, encargado de la operación del equipo. Para comparar y tratar de identificar los materiales pétreos en que estaban hechas las estelas se usaron muestras geológicas de referencia que forman parte de los muestrarios de rocas y minerales del Museo del Templo Mayor y del Laboratorio de Geología de la Escuela Nacional de Conservación, Restauración y Museografía (ENCRyM) del INAH, las cuales han sido caracterizadas anteriormente por medio de petrografía con láminas delgadas y difracción de rayos X. Así fue posible realizar la identificación sobre la materia prima en la cual fueron manufacturadas las estelas (Fig. 5).

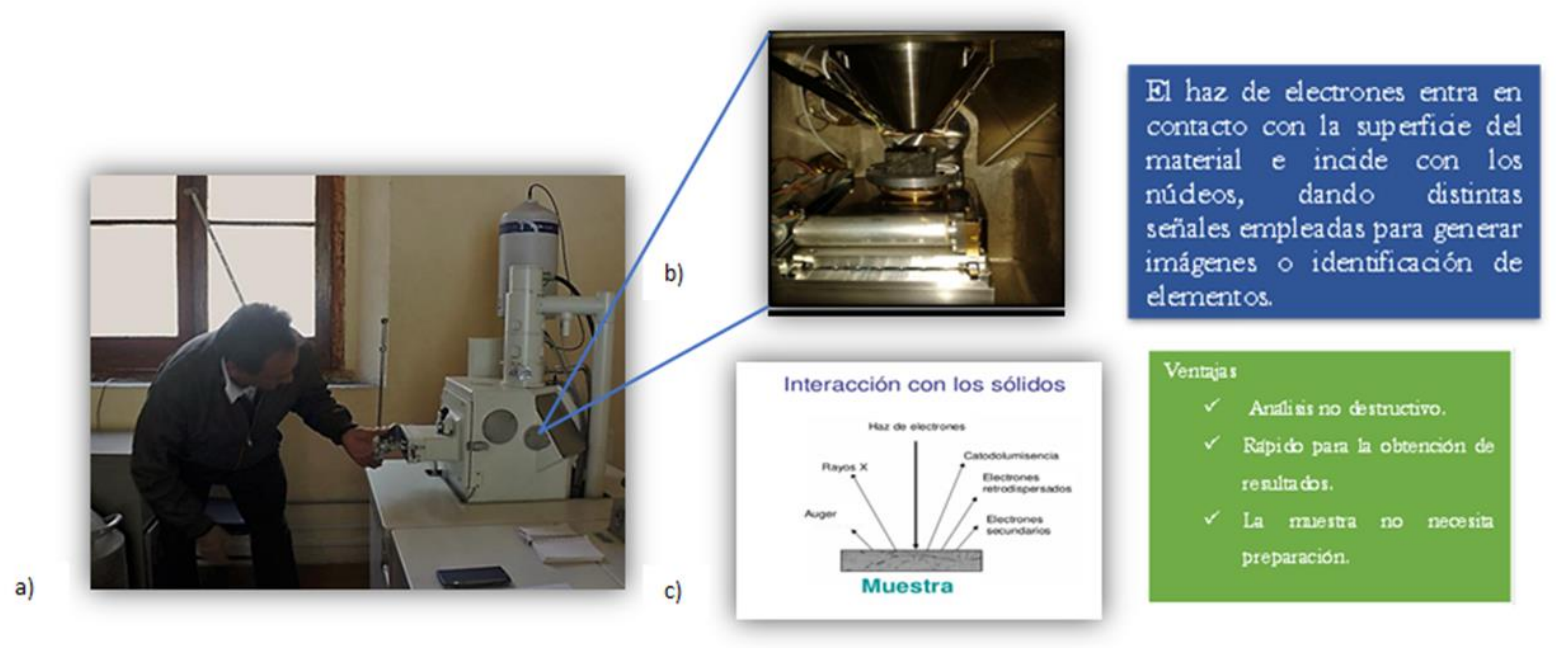

Figura 4. a) Ingeniero Mario Monroy metiendo muestras en el EDS (Fotografía de Viridiana Guzmán, 2017), b) Interior del EDS con muestra (Fotografía de Emiliano Melgar, 2017), c) Esquema de funcionamiento del EDS. 
a)

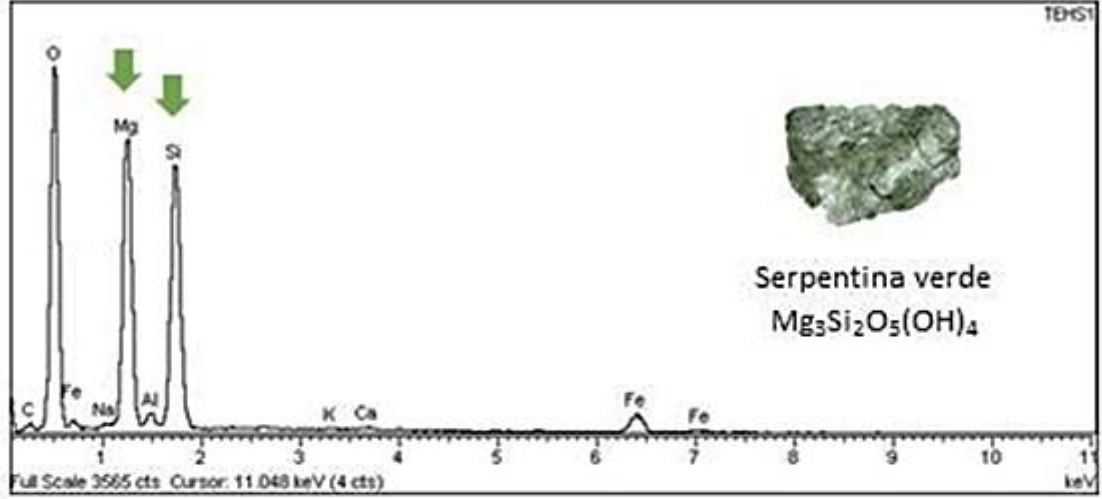

b)

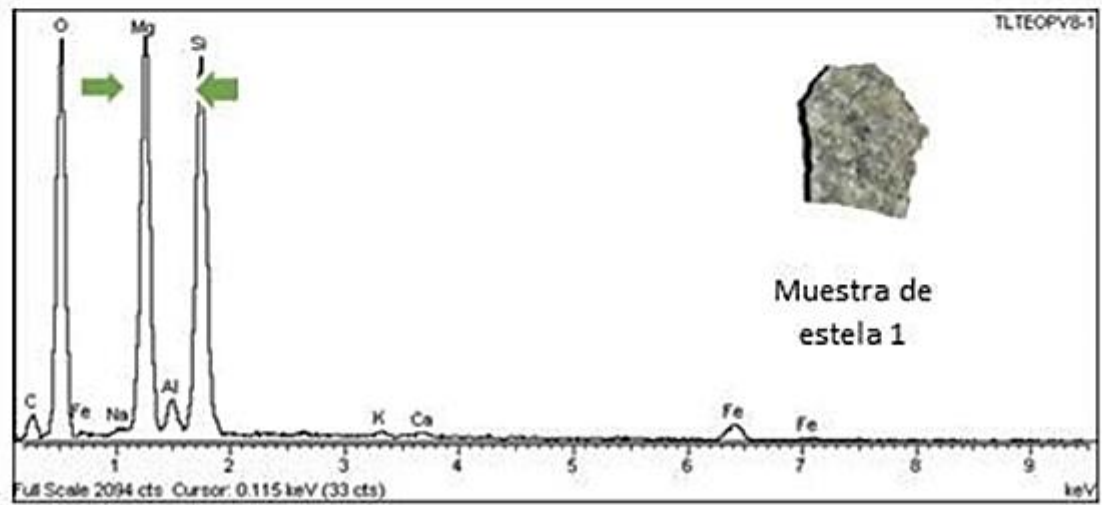

c)

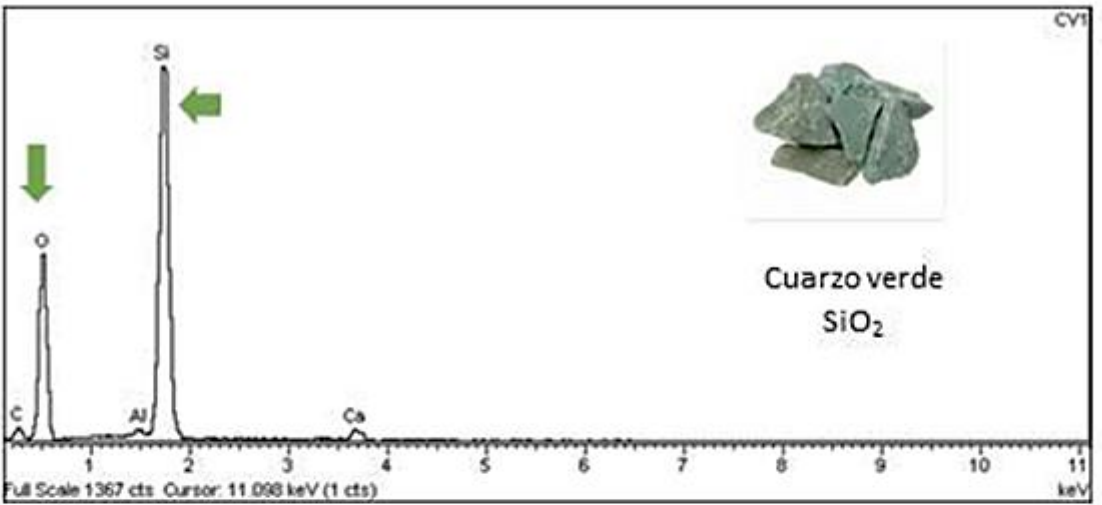

d)

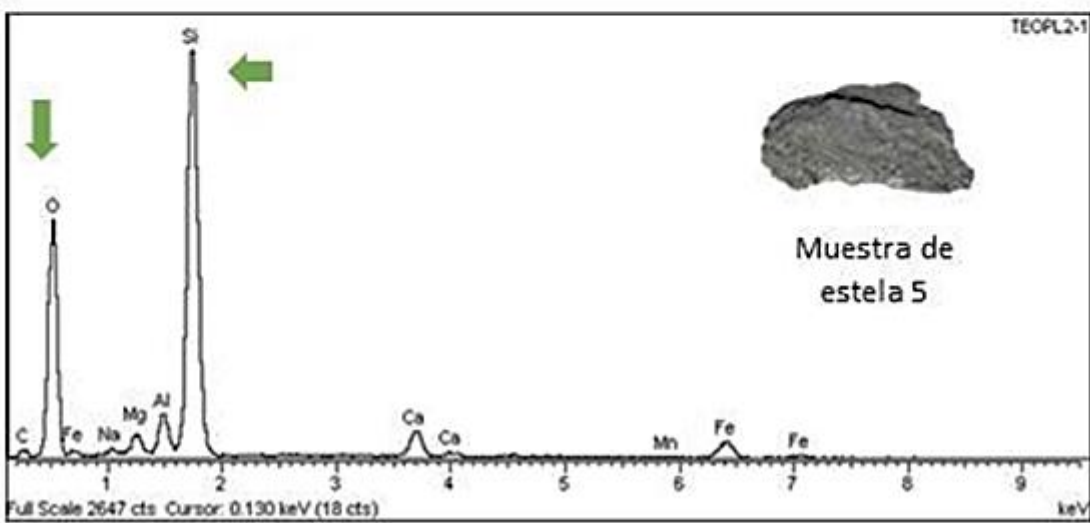

Figura 5. Microsonda de Rayos X. a) Espectro de muestra de serpentina, b) Muestra de Estela 1, c) Espectro de muestra de cuarzo y d) Muestra de Estela 5 (Espectros procesados por Emiliano Melgar y Mario Monroy) (Fotografías de Emiliano Melgar). 


\subsection{Espectrometría de micro-RAMAN ( $\mu$ Raman)}

El análisis de composición química elemental con EDS se complementa con la Espectroscopía Micro-Raman ( $\mu$ Raman), la cual permite determinar la presencia de un mineral o compuesto en general a partir de un haz de láser verde (de $532 \mathrm{~nm}$ ) que interactúa con las muestras, cuya dispersión inelástica de fotones, llamada efecto Raman en honor a su descubridor, el hindú Venkata Raman, produce espectros vibracionales a partir de una emisión luminosa característica de los materiales que integran la región analizada (FEUM, 2011:340). Para esta técnica se empleó el equipo de $\mu$ Raman, modelo Thermo Scientific DXR, del Laboratorio de Materiales Avanzados del Instituto de Física de la UNAM, con apoyo de la Dra. Cristina Zorrilla Cangas (Figura 4). Todas las piezas se observaron a 20 y 50x, apertura de $50 \mu \mathrm{m}$ slit, $6.6 \mathrm{~mW}$, con un intervalo espectral de 0 a $3500 \mathrm{~cm}^{-1}$ y un tiempo de adquisición de 10 repeticiones de 10 segundos. Los espectros obtenidos se procesaron con el programa OMNIC®. De tal manera, al someter los materiales a la luz infrarroja fue posible observar opacidades o reflejos (Fig. 6), que son causadas por compuestos y enlaces moleculares de determinados minerales (Ruvalcaba, et. al. 2010:156). Lo anterior nos ayudó a comprender con mayor claridad la naturaleza mineralógica del material y hacer comparaciones del espectro Raman de cada estela con el muestrario de rocas y minerales, detectando que la mayoría de las estelas compartían varios picos con serpentinas y una con cuarzos verdes (Fig. 7).

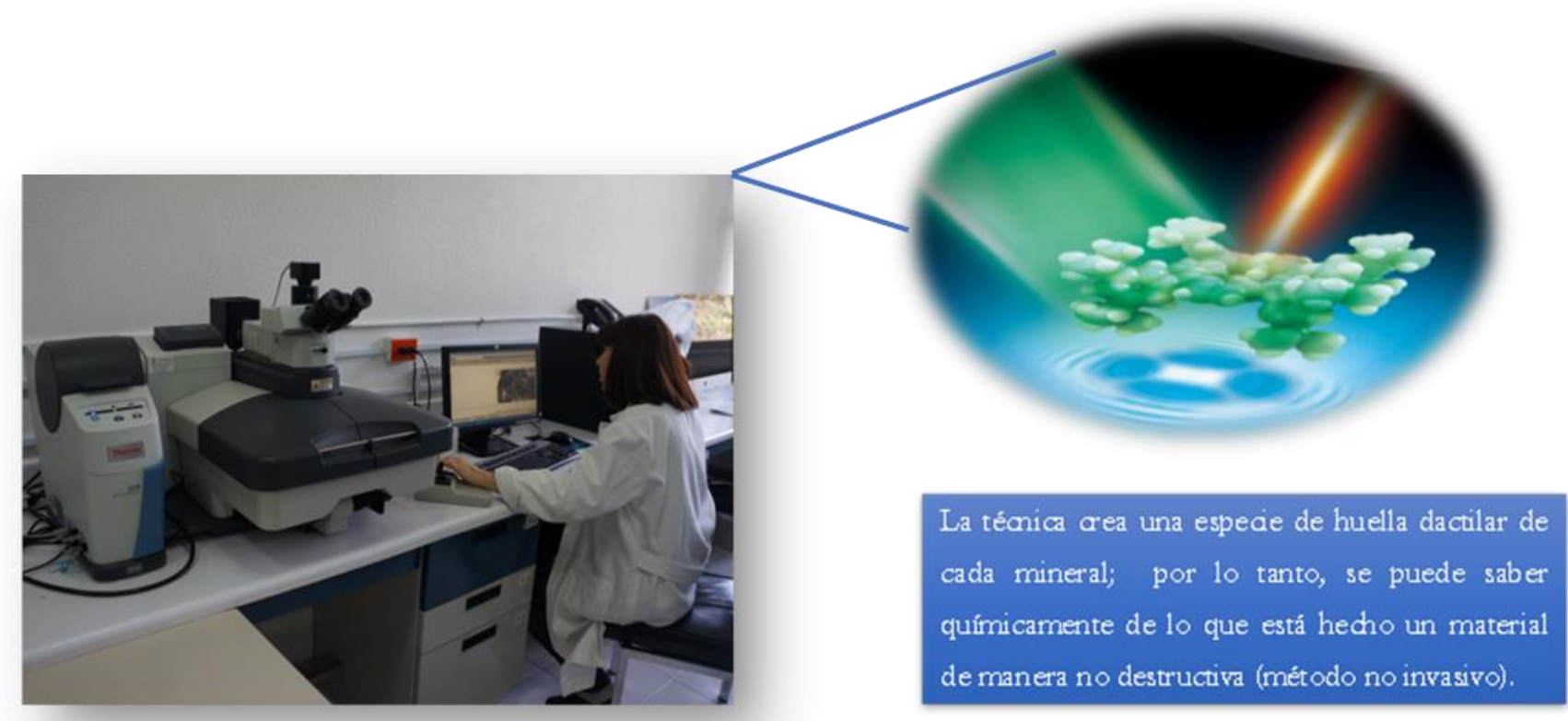

Figura 6. Doctora Cristina Zorrilla manejando Micro-RAMAN ( $\mu$ Raman) en IF-UNAM (Fotografía de Guzmán, 2017). 

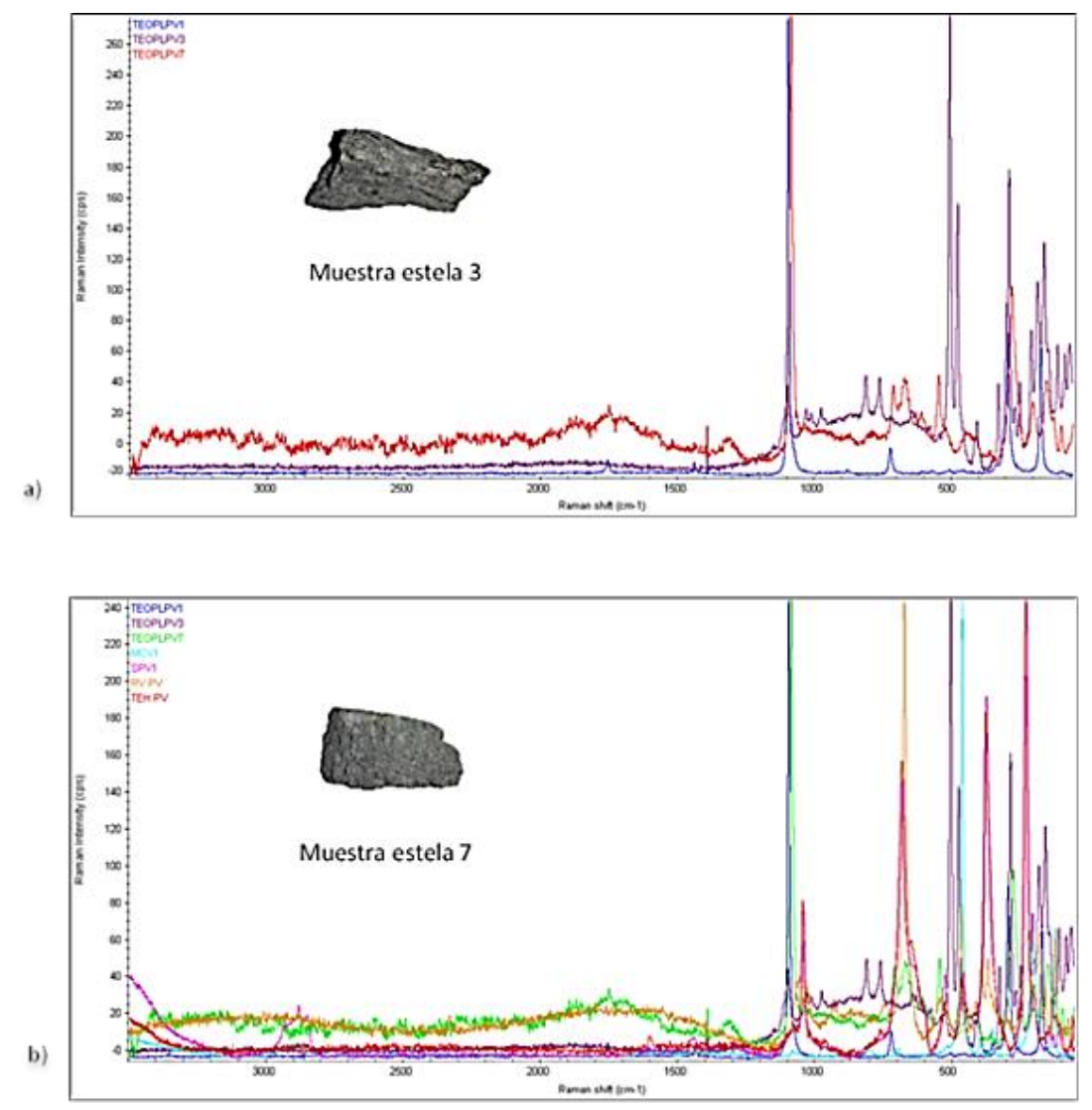

Figura 7. Micro -Raman, a) Espectros de 3 Estelas y b) Empalme de espectros y muestras de referencia. (Espectros procesados por Emiliano Melgar y Cristina Zorrilla) (Fotografías de Emiliano Melgar).

\subsection{Análisis macroscópico}

Consistió en la observación a simple vista de las modificaciones de los monolitos tales como la regularidad de la superficie: borde, relieve, presencia de líneas y desgastes.

\section{PROCESO EXPERIMENTAL}

La experimentación, como método de análisis en arqueología, permite reconstruir los procesos por los que pasaron para ser manufacturados los objetos encontrados en distintos contextos arqueológicos (Fig. 8). Teniendo en consideración lo anterior se reprodujeron experimentalmente las modificaciones observadas a simple vista de los monolitos, sin embargo, la revisión de los mismos a un nivel macroscópico no es suficiente, debido a que no es posible determinar con ello los instrumentos utilizados para la manufactura de los objetos. De tal forma que se realizaron posteriormente las observaciones de las modificaciones por medio de microscopía electrónica de barrido (SEM). 


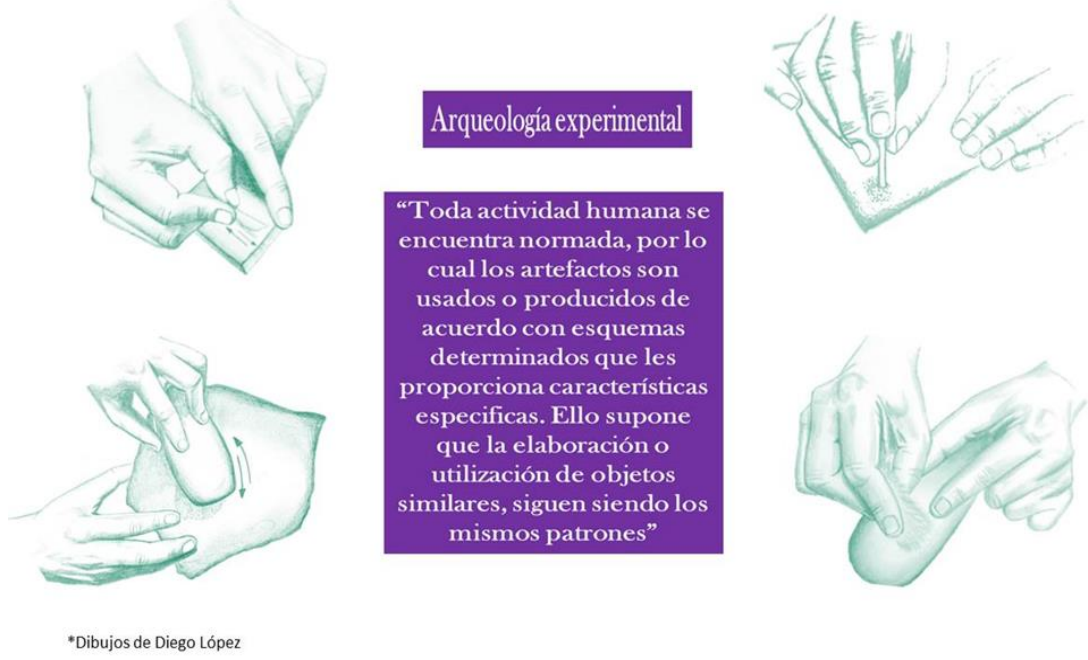

Figura 8. Dibujos de arqueología experimental. (Dibujos de Diego López).

\subsection{Microscopía Electrónica de Barrido (SEM)}

Se obtuvieron réplicas de las distintas modificaciones encontradas en las estelas a través de un molde que fue tomado con una cinta especializada de polímero (cinta de polímero replicante marca Buehler), la cual, al agregarle unas gotas de acetona se adhirió a la superficie del material. Posteriormente se llevaron al Microscopio Electrónico de Barrido (SEM, por sus siglas en inglés) (modelo Jeol JSM-6460LV), donde se les realizó un recubrimiento con iones de oro (Sputtering) y se empleó el SEM en modo de alto vacío a 100x, 300x, 600x y 1000x. Las condiciones de trabajo fueron utilizadas a la misma frecuencia de electrones secundarios (SEI), tamaño de haz (42), distancia de trabajo (10 $\mathrm{mm})$ y voltaje $(20 \mathrm{kV})$, que generó una imagen detallada de las modificaciones, con lo cual fueron visibles las marcas generadas por las herramientas de trabajo a las que se les denomina huellas de manufactura (Fig. 9).
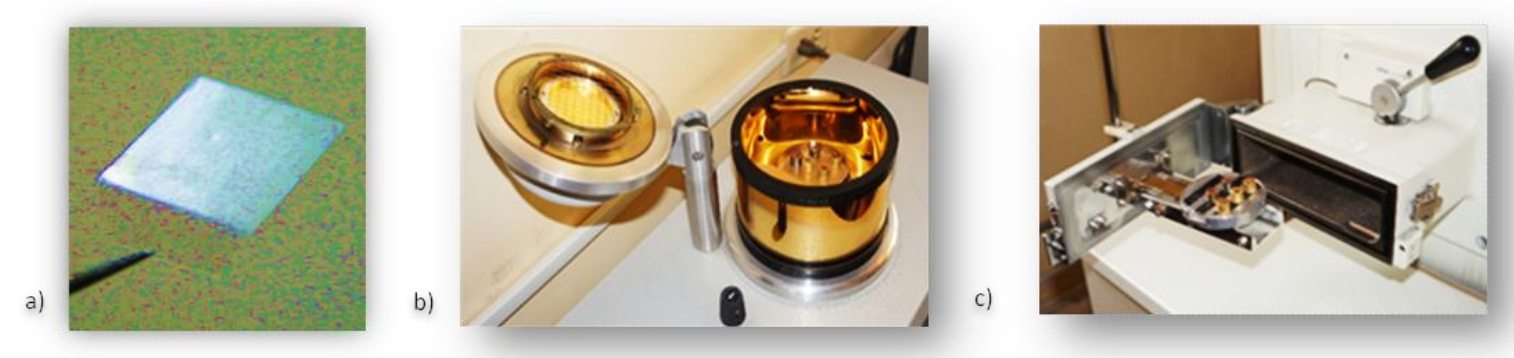

Figura 9. A) Réplica en polímero, b) Recubrimiento con iones de oro, y c) Muestras ingresando al SEM (Fotografía de Viridiana Guzmán, 2017).

La caracterización de las huellas de manufactura en los objetos nos permite inferir la tecnología empleada e identificar los estilos y tradiciones lapidarias llevadas a cabo por los diversos grupos prehispánicos (Melgar, 2014: 14).

Al realizar una comparación entre las huellas de materiales arqueológicos y las producidas experimentalmente se puede confirmar; o, en su defecto, refutar las herramientas propuestas para la manufactura de dichos materiales (Tabla 1). 


\begin{tabular}{|c|c|}
\hline \multicolumn{2}{|c|}{ Rasgos visibles empleando Microscopía Electrónica de Barrido } \\
\hline Rasgo visible & Descripción \\
\hline Banda & Franjas rectas que van de 2-100 $\mu$ m de grosor. \\
\hline Lineas & Rayas rectas menores de $2 \mu \mathrm{m}$ de grosor. \\
\hline Líneas difusas & Rayas rectas atenuadas y poco perceptibles. \\
\hline Partículas & 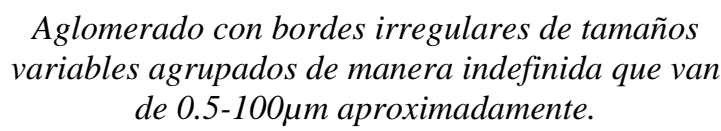 \\
\hline $\begin{array}{l}\text { Superficie } \\
\text { aplanada }\end{array}$ & $\begin{array}{c}\text { Superficie regular, aplanada con rasgos de } \\
\text { anteriores modificaciones difusos (ligeramente } \\
\text { perceptibles). }\end{array}$ \\
\hline $\begin{array}{l}\text { Superficie } \\
\text { irregular }\end{array}$ & Superficie con zonas aplanadas e irregulares. \\
\hline Superficie lisa & Superficie regular y aplanada. \\
\hline Superficie rugosa & $\begin{array}{c}\text { Superficie irregular, con pliegues similares a } \\
\text { hojuelas. }\end{array}$ \\
\hline
\end{tabular}

El estudio macroscópico de los monolitos hallados en el subsuelo de la Estructura A, se observó que las superficies tienen evidencias de adelgazamiento o aplanamiento que sugiere el uso de materiales líticos como lajas elaboradas en rocas. Experimentalmente, para realizar la caracterización de huellas de manufactura, fue necesario utilizar metates de andesita y basalto (por mencionar algunos materiales empleados durante la experimentación) y en algunos casos, se agregaron abrasivos como arena. El procedimiento consistió en frotar las materias primas con movimientos de vaivén alterno sobre las lajas o metates de roca sin abrasivo; posteriormente se realizaron los experimentos con abrasivos, humedeciéndolo y remplazándolo, por cierto tiempo, debido a que se formaba una pasta que hacía que el abrasivo perdiera su efectividad después de

a)
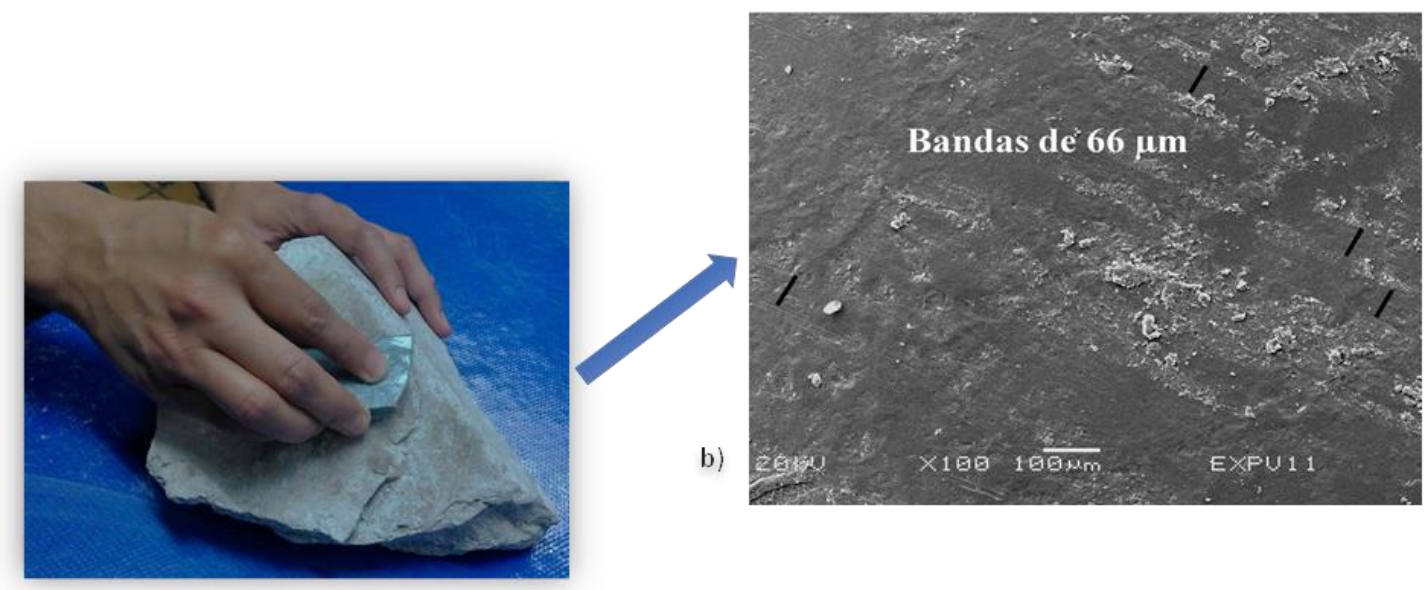

Tiempo: 30 minutos

Figura 10. A) Desgaste experimental con andesita (Fotografía Viridiana Guzmán), b) y micrografía a 100x (Micrografía analizada por Emiliano Melqar). 
V. Ortega, V. Guzmán, E. R. Melgar y S. Sánchez

Análisis tecnológico de los monolitos de piedra verde hallados en la Plaza de la Pirámide de la Luna, Teotihuacán

un período de tiempo de uso. En los experimentos realizados se utilizó regularmente agua para enfriar los materiales, debido a la fricción generada en el desgaste (Fig. 10).

Se llevaron a cabo los experimentos de desgaste por un tiempo determinado de trabajo de media hora para obtener huellas suficientemente marcadas y poderlas observar. Los rasgos que surgieron después de la experimentación permitieron apreciar que los materiales desgastados sin abrasivos dejan rayones rectos marcados sobre la superficie; mientras que en los que se utilizaron abrasivos se observan superficies de textura regular, apariencia brillante, suave y tersa, con líneas finas y difusas (Melgar, 2014:242).

Los monolitos presentaron una apariencia lisa y brillante, por lo cual, se plantea que les fue aplicado algún acabado, provocando que los desgastes iniciales se hicieran difusos.

Los experimentos fueron realizados por medio de tres técnicas; pulido, bruñido y la combinación de los dos anteriores, sobre superficies previamente desgastadas con lajas o metates, con y sin abrasivos (Fig. 11).

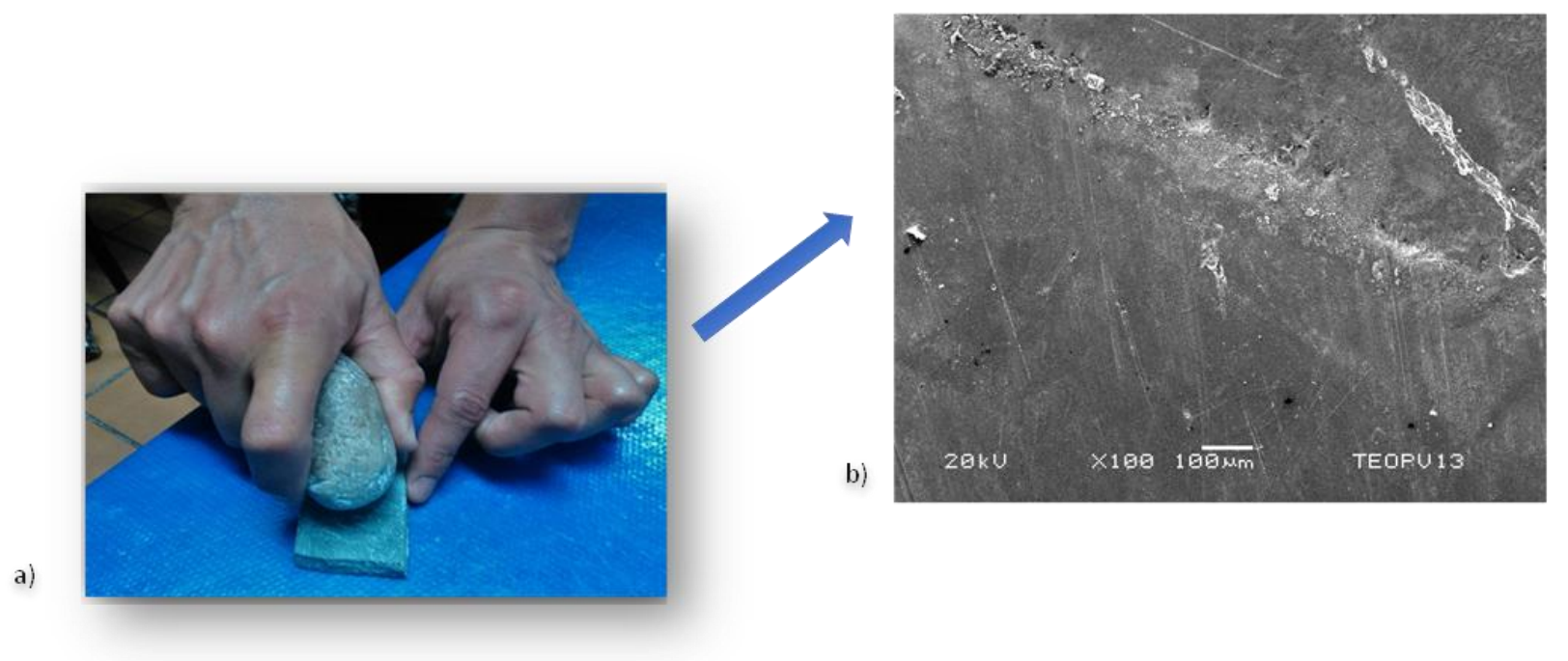

\section{Tiempo: 30 minutos}

Figura 11. a) Pulido experimental con nódulo de pedernal sobre serpentina (Fotografía Viridiana Guzmán), b) y micrografía a 1000x (Micrografía analizada por Emiliano Melgar).

El pulido se obtuvo por medio de aplicación de arena humedecida y un trozo de piel, el cual se frotó contra una superficie desgastada con movimientos de vaivén alterno y con herramientas líticas como el pedernal.

Se hicieron bruñidos mediante el empleo de trozos de piel en seco que se frotaron sobre superficies desgastadas, con movimientos de vaivén alterno y con movimientos rotatorios. 
Las huellas obtenidas por medio del desgate experimental con una laja de andesita y serpentina fueron bandas de entre 2 a $4 \mu \mathrm{m}$ y bandas entrecruzadas de aproximadamente 66 a $70 \mu \mathrm{m}$, las cuales al ser comparadas con los polímeros replicantes obtenidos de las estelas coinciden con el tipo de huella (Fig. 12).
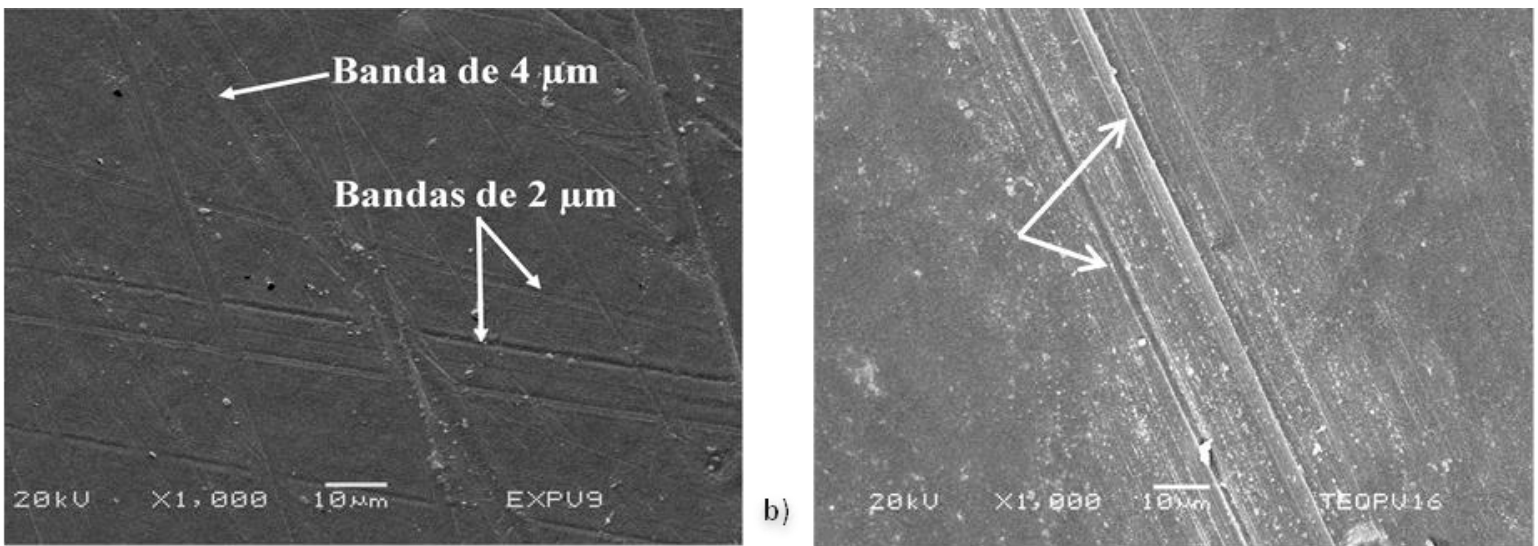

Figura 12. a) Micrografía de superficie de experimental a 1000x, b) y micrografía de estela 1000x (Micrografías analizadas por Emiliano Melgar).

En cuanto el acabado de superficie, fue posible caracterizar el pulido experimental por medio de nódulos de pedernal sobre una serpentina, fueron bandas de entre 2 a $4 \mu \mathrm{m}$ con microrrayados internos aplanados y difusas debido a que la piel alisa los rasgos que al ser contrastadas con las micrografías de las estelas comparten los rasgos característicos de la aplicación de pedernal y piel. Finalmente se puede apreciar la coincidencia, en cuanto a tecnología, de las huellas obtenidas experimentalmente en estos objetos con otros similares encontrados en la urbe de Teotihuacán (Fig. 13).
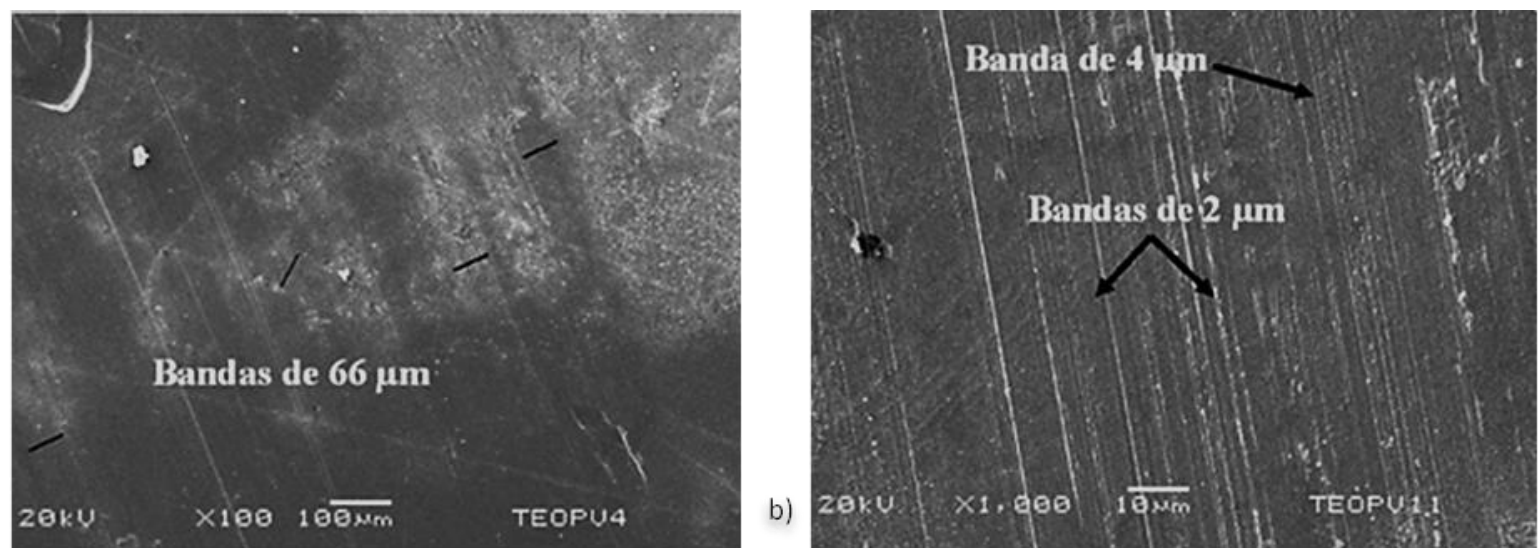

Figura 13. a) Micrografía de superficie Placa trapezoidal de Teopancazco a 100x, b) y micrografía a 1000x (Micrografías analizadas por Emiliano Melgar).

\section{RESULTADOS}

Por medido de la arqueología experimental y el análisis de huellas de manufactura, fue posible identificar las herramientas utilizadas para la elaboración de los monolitos encontrados en la Estructura A, en Plaza de la Pirámide de la Luna. Por otro lado, gracias a las técnicas de identificación de composición elemental (EDS) y molecular ( $\mu$ Raman), permitió conocer la naturaleza de la materia prima sobre la cual fueron manufacturadas 
V. Ortega, V. Guzmán, E. R. Melgar y S. Sánchez

Análisis tecnológico de los monolitos de piedra verde hallados en la Plaza de la Pirámide de la Luna, Teotihuacán

las estelas. Finalmente, en conjunto, los análisis tecnológicos y de composición permitieron realizar una filiación cultural de las mismas.

El análisis EDS, arrojó que la composición estos objetos es diversa; son piedras verdes metamórficas con variaciones en sus elementos coincidiendo con lo detectado en el $\mu$ Raman el cual indicó, al ser comparadas las muestras de las estelas con serpentinas y otros aluminosilicatos de referencias geológicas conocidas, tener el pico indicados del cuarzo y una cercanía con las serpentinas. El monolito 1 es un silicato de magnesio debido al alto nivel de magnesio es parecida a una serpentina. El monolito 2 es un aluminosilicato rico en magnesio, hierro y calcio, cercana a una serpentina pero no perfecta en su composición. El monolito 3 es un aluminosilicato rico en magnesio y hierro, es cercana a una serpentina pero no perfecta en su composición. El monolito 5 es la más rica en cuarzo al tener los niveles altos de óxido de silicio, mientras que el magnesio y de más elementos son muy bajos.

En cuanto a la arqueología experimental y al análisis tecnológico de la manufactura de los monolitos, se observó que las modificaciones obtenidas con andesita para desgastar la superficie y pedernal para pulir, vistas en el Microscopio Electrónico de Barrido (SEM), al ser comparadas las huellas de manufactura, experimentales ya arqueológicas, fue visible la coincidencia en cuanto a la medida y forma de la huellas, además de coincidir con la manufactura de otras piezas encontradas en el área de Teotihuacan (como son placas trapezoidales y las narigueras en forma de mariposa), por lo que podríamos inferir que la manufactura de los monolitos encontrados en las fosas de la Estructura A, Plaza de la Pirámide de la Luna, fue local.

Aún quedan cuestiones por resolver, pues si bien se logró identificar la materia prima de los monolitos, aún no se cuenta con la identificación del yacimiento del cual provienen. Finalmente es necesario mencionar que las muestras obtenidas de estos objetos actualmente están siendo analizadas por medio de otros estudios como petrografía, para identificar con mayor precisión la estructura de la roca y el origen de la misma.

\section{Agradecimientos}

La investigación fue posible gracias al apoyo del Ingeniero Mario Monroy Escamilla, del Laboratorio de Microscopía Electrónica de Barrido (SEM) del INAH, Cristina Zorrilla, del Laboratorio de Materiales Avanzados del IF-UNAM y de los integrantes del "Taller de arqueología experimental en lapidaria" del Museo Templo Mayor, Reyna Solís, Andrea Pérez, Adriana Soto, Marcos Reyes, Edgar Pineda, Henri Bernard, Juan Carlos Meléndez y Hervé Monterrosa, y el apoyo de Rodrigo Vega.

\section{Bibliografía}

FEUM (2011): [Sitio Web] https://www.farmacopea.org.mx/ [Fecha de último acceso 17/05/2019] Comisión permanente de la Farmacopea de los Estados Unidos Mexicanos. México: Secretaría de Salud.

GRAULICH, M. (2014): Moctezuma: Apogeo y caída del imperio azteca, Ediciones Era, México.

GAZZOLA, J. (2009): "La cadena operativa en la fabricación de máscaras en los talleres de lapidaria de La Ventilla, Teotihuacan". En MIRAMBELL, L. y GONZÁLEZ ARRATIA, L. (eds.): Investigaciones recientes sobre la lítica arqueológica, pp. 61-77, Colección científica 561, INAH. 
KYUNG KANG, W., ECHARRI, J. M. y LLORENTE, C. L. (2013): "Servicio de microscopía electrónica de barrido y microanálisis del LIMF-Facultad de Ingeniería. UNLP". Conferencia impartida en las Segundas Jornadas de Investigación y transferencia, Departamento de Mecánica, Universidad Nacional de La Plata. [Online] http://sedici.unlp.edu.ar/handle/10915/38238 [Fecha de último acceso 17/05/2019].

MANZANILLA, L. R. (2012): Estudios arqueométricos del centro de barrio de Teopancazco en Teotihuacan, UNAM, México.

MELGAR TÍSOC, E. R. (2014): Comercio, tributo y producción de las turquesas del Templo Mayor de Tenochtitlan. México. Tesis de Doctorado en Antropología, UNAM.

MELGAR TÍSOC, EMILIANO R., SOLÍS CIRIACO, R. B., y RUVALCABA SIL, J. L. (2012): "La lapidaria de Teopancazco: Composición y manufactura". En MANZANILLA, L. R. (ed.): Estudios arqueométricos del centro de barrio de Teopancazco en Teotihuacan, pp. 247-284, UNAM, México.

ORTEGA CABRERA, V. (2014): Informe técnico del Proyecto de Conservación Integral del Edificio 1, Plaza de la Luna, Teotihuacán. Documento entregado al Archivo Técnico de la Coordinación Nacional de Arqueología, INAH, México

ORTEGA CABRERA, V. (2015): Informe técnico del Proyecto de Conservación Integral de la Estructura A, Plaza de la Luna, Teotihuacán. Documento entregado al Archivo Técnico de la Coordinación Nacional de Arqueología, INAH, México.

RATTRAY, E. C. (1997): Entierros y ofrendas en Teotihuacan: Excavaciones, inventario, patrones mortuorios.: Instituto de Investigaciones antropológicas UNAM, México.

RUVALCABA-SIL, J. L., FILLOY, L., VAGGI, M., TAPIA-GÁLVEZ, L. H. у SÁNCHEZ-BECERRA, R. (2010): "Estudio no destructivo in situ de la Máscara de Malinaltepec". En MARTÍNEZ DEL CAMPO, S. (ed.): La Máscara de Malinaltepec, pp. 153-168, CONACULTA-INAH, México.

SOLÍS CIRIACO, R. B. (2015): Esferas de producción y consumo de la lapidaria de los edificios aledaños al Templo Mayor de Tenochtitlan, México. Tesis de Doctorado en Antropología, UNAM, México. 